\title{
Huella del Carbono. Parte 1: Conceptos, Métodos de Estimación y Complejidades Metodológicas
}

\author{
César Espíndola ${ }^{(1,2)}$ y José O. Valderrama ${ }^{(3,4)}$ \\ (1) Univ. de La Serena, Fac. de Ingeniería, Dpto. de Ing. Industrial, Casilla 554, La Serena-Chile \\ (2) Univ. de Lleida, Depto. Adm. Empresas y Gestión Económica de Rec. Naturales, Lleida-España \\ (3) Univ. de La Serena, Fac. de Ingeniería, Dpto. de Ing. Mecánica., Casilla 554, La Serena-Chile \\ (4) Centro de Información Tecnológica (CIT), Casilla 724, La Serena-Chile
}

Recibido Sep. 10, 2011; Aceptado Oct. 25, 2011; Versión Final recibida Dic. 02, 2011

\section{Resumen}

Se presenta y analiza el concepto de huella del carbono, su origen, su relación con los gases efecto invernadero, y sobre los procedimientos para cuantificarla. El efecto invernadero provoca que la energía que llega a la Tierra sea devuelta más lentamente, por lo que es mantenida más tiempo junto a la superficie elevando la temperatura. Es aceptado hoy en día que este efecto es producido por algunos gases liberados en forma natural o por las acciones humanas. La Huella de Carbono es considerada una de las más importantes herramientas para cuantificar las emisiones de gases efecto invernadero y en forma muy general, representa la cantidad de gases efecto invernadero emitidos a la atmósfera derivados de las actividades de producción o consumo de bienes y servicios. Al no existir consenso en cuanto a la definición y menos en la cuantificación de la huella del carbono, la primera parte de este trabajo analiza las principales corrientes y enfoques actuales. global

\section{Carbon Footprint. Part 1: Concepts, Estimation Methods and Methodological Complexities}

\begin{abstract}
The concept of Carbon Footprint, its origin, its relation with greenhouse gases and the methods to quantify it are presented and analyzed. The so-called greenhouse effect causes that the energy that reaches the earth at a certain rate is returned to a slower rate, increasing the temperature of the earth surface. Additionally, it is nowadays accepted that this effect is produced by some gases that are naturally emitted or produced by human actions. The Carbon Footprint is considered to be one of the most important tools for quantifying greenhouse emissions and in a general form it represents the quantity of gases emitted to the atmosphere and that is produced by human activities, and by goods and service consumption. Since there is no consensus about the definition of Carbon Footprint or the forms of quantifying it, this first part of the paper series analyzes the main concept and the main present views on the Carbon Footprint.
\end{abstract}

Keywords: carbon footprint, greenhouse gases, climate change, global warming 


\section{INTRODUCCIÓN}

La Huella de Carbono (HdC), definida en forma muy general, representa la cantidad de gases efecto invernadero (GEI) emitidos a la atmósfera derivados de las actividades de producción o consumo de bienes y servicios (Pandey et al., 2010; Wiedmann, 2009), y es considerada una de las más importantes herramientas para cuantificar las emisiones de dichos gases. Los GEI, definidos en el protocolo de Kioto el año 1997, forman una capa permanente en la parte media de la atmósfera que impide que toda la radiación solar que es devuelta por la tierra pueda salir, provocando con ello que la temperatura bajo la capa aumente.

En los últimos años se han desarrollado varias otras herramientas de cuantificación y metodologías para determinar el nivel de emisiones de GEI de individuos, organizaciones y unidades administrativas o territoriales, y la HdC es una de ellas (Padgett et al., 2008). La HdC, se ha convertido en un lema en el debate público sobre el cambio climático, atrayendo la atención de los consumidores, negocios, gobiernos, ONG y organizaciones internacionales por igual (Hertwich y Peters, 2009), induciendo cambios en los patrones competitivos de las empresas. A pesar de su uso generalizado, la literatura reconoce la ausencia hasta hoy en día de una clara definición que sea comúnmente aceptada (Wiedmann y Minx, 2008). Este debate sobre el cambio climático y la utilidad de $\mathrm{HdC}$, ha trascendido al comercio internacional $\mathrm{y}$, es liderado por los países con compromisos de reducción de emisiones en base al Protocolo de Kioto. (Plassmann et al., 2010) Esto es motivado fundamentalmente por la preocupación de estos países por las posibles pérdidas de competitividad de sus productores, quienes estarían compitiendo con otros exportadores con costos de emisión menores que aquellos que no han asumido obligaciones climáticas (De La Torre et al., 2009)

En el caso de los países en vías de desarrollo, como es el caso de la mayoría de los países latinoamericanos, un patrón exportador más acorde con las aspiraciones de desarrollo sostenible y menos vulnerable a las exigencias climáticas frente a la instalación de un concepto económico que considera la reducción de las emisiones contaminantes, exige a los sectores productivos realizar avances inmediatos en los proceso de cuantificación de sus emisiones y de disminución de los efectos climáticos, con el fin de resguardar su actual posición competitiva.(Schneider y Samaniego, 2009; CEPAL 2009). Sin embargo, se hace evidente que previo a la cuantificación, se requiere explorar las complejidades metodológicas de los principales enfoques para la determinación de la $\mathrm{HdC}$, con el objeto de reducir la incertidumbre del cálculo y determinar su validez en la determinación de los reales impactos ambientales.

Un número creciente de grupos sociales, empresariales y políticos de los más diversos países y una parte importante de la comunidad científica están convencidos de que el cambio climático es originado por las actividades humanas y que constituye, por lo tanto, uno de los mayores desafíos ambientales que se pudiera interponer en el camino hacia el desarrollo sustentable en el presente siglo (WRI, 2004). Así también, es ampliamente aceptado que la causa de dicho fenómeno se encontraría en las altas concentraciones atmosféricas de los llamados "gases de efecto invernadero" (GEI), las cuales serían responsables de aumentar la temperatura global del planeta (Pachauri y Reisinger, 2007). El Panel Intergubernamental sobre Cambio Climático (IPCC) perteneciente al Programa de Medio Ambiente de las Naciones Unidas y la Organización Mundial Meteorológica, ha indicado que el riesgo del cambio climático es severo y que su impacto aumentará notablemente con un incremento de las temperaturas en $2^{\circ} \mathrm{C}$ por encima de las registradas en la época preindustrial (EPA, 2011). Aparte de ser un problema ambiental, el cambio climático constituye un problema de desarrollo, con profundos impactos potenciales en la sociedad, la economía y los ecosistemas. (Stern, 2006; Pandey et al., 2010). Esto ha llevado a gobiernos e instituciones internacionales a implementar una serie de acciones, tales como la creación de órganos a escala nacional e internacional preocupados del tema, la definición de procesos y espacios de reflexión, la creación de herramientas de transferencia tecnológica y financiera, que ayuden a la mitigación de emisiones de GEI provenientes de la actividad humana y a la formulación de métodos para cuantificar los efectos de dichos gases (WRI, 2004; WBCSD, 2004). 


\section{GASES EFECTO INVERNADERO}

El efecto invernadero se origina porque la energía que llega del sol está formada por ondas de frecuencias altas que traspasan la atmósfera, sin mucha resistencia. La energía remitida hacia el exterior, desde la Tierra está formada por ondas de frecuencias más bajas, y es absorbida por los gases, produciendo el efecto invernadero. Esta retención de la energía hace que la temperatura aumente. En forma simple el efecto invernadero provoca que la energía que llega a la Tierra sea devuelta más lentamente, por lo que es mantenida más tiempo junto a la superficie elevando la temperatura (Bolin et al., 1986). El rápido incremento de la temperatura global es producto del "efecto invernadero", debido a la liberación de GEI de origen antropogénico a la atmósfera. No todos los GEI tienen la misma capacidad de provocar calentamiento global, pero su intensidad depende de su poder de radiación y el tiempo promedio que la molécula del gas permanece en la atmósfera. Si estos dos factores se consideran juntos, al promedio de calentamiento que pueden causar, se le conoce como "Potencial de Calentamiento Global" o PCG por sus siglas en inglés, el cual es obtenido matemáticamente y es expresado en relación con el nivel de $\left(\mathrm{CO}_{2}\right)$, es decir, el PCG tiene por unidad al dióxido de carbono equivalente $\left(\mathrm{CO}_{2}-\mathrm{e}\right)$. La Tabla 1 muestra la contribución al calentamiento global de varios gases contaminantes y efecto invernadero.

Como se observa en la Tabla 1 en la columna de acción relativa, un gramo de CFC13 produce un efecto invernadero de 14 mil veces más que lo que produce un gramo de $\mathrm{CO}_{2}$, pero como la cantidad de $\mathrm{CO}_{2}$ es mucho mayor que la del resto de los gases, la contribución real del $\mathrm{CO}_{2}$ al efecto invernadero resulta mayor. Se puede ver también por ejemplo que el metano $\left(\mathrm{CH}_{4}\right)$ y el refrigerante $134 \mathrm{a}$, con una vida media parecida (12 y 14 años respectivamente) difieren grandemente en el potencial como GEl de 25 para el $\mathrm{CH}_{4}$ a 1430 para el refrigerante 134a $\left(\mathrm{CH}_{2} \mathrm{FCF}_{3}\right)$. Esto es peor para el hexafluoruro de fósforo $\left(\mathrm{SF}_{6}\right)$ que con una vida media de 3200 años tiene un potencial como GEI de 22800. En el último siglo la concentración de GEI en la atmósfera ha ido creciendo constantemente debido a la actividad humana (BSI, 2008).

Hay consenso general de que a mayor concentración de gases con efecto invernadero se producirá mayor aumento en la temperatura en la Tierra. A partir de 1979 los científicos comenzaron a afirmar que un aumento al doble en la concentración del $\mathrm{CO}_{2}$ en la atmósfera supondría un calentamiento medio de la superficie de la Tierra de entre 1.5 y $4.5^{\circ} \mathrm{C}$. Estudios más recientes sugieren que el calentamiento se produciría más rápidamente sobre tierra firme que sobre los mares. Al principio los océanos más fríos tenderán a absorber una gran parte del calor adicional retrasando el calentamiento de la atmósfera. Sólo cuando los océanos lleguen a un nivel de equilibrio con los más altos niveles de $\mathrm{CO}_{2}$ se producirá el calentamiento final. Como consecuencia del retraso del efecto de los gases en la temperatura de los océanos más fríos, los científicos no esperan que la Tierra se caliente más de $4^{\circ} \mathrm{C}$ como se había previsto hace un tiempo, incluso si el nivel de $\mathrm{CO}_{2}$ subiera a más del doble y se añadieran otros gases con efecto invernadero. En la actualidad el IPCC predice un calentamiento de 1.0 a $3.5^{\circ} \mathrm{C}$ para el año 2100 (IPCC, 2011).

\section{CUANTIFICACIÓN DE LA HUELLA DEL CARBONO}

Como se menciona más arriba, la HdC sólo ha surgido en el dominio público en los últimos años como una descripción bastante general de la emisión de gases de efecto invernadero totales asociados con la actividad humana. La literatura reconoce la ausencia de una clara definición que sea comúnmente aceptada (Wiedmann y Minx, 2008). Sin embargo, se encuentran antecedentes de marcos metodológicos utilizados para cálculo de la HdC desde los años 70 del siglo pasado (Daly, 1968; Leontief, 1970; Leontief y Ford, 1971; Victor, 1972). Desde fines de los años 1980 se encuentran en la literatura aplicaciones de la HdC, aunque bajo nombres diferentes (Minx et al., 2010). Desde entonces, el debate sobre la Huella del Carbono no sólo alcanza a su origen sino también al enfoque metodológico empleado en su análisis. En la academia el tema ha experimentado también un notable desarrollo. Desde unas pocas publicaciones a fines del siglo pasado la literatura relacionada con la $\mathrm{HdC}$ ha experimentado un crecimiento exponencial en los últimos 3 años (Valderrama et al., 2011). 
Tabla 1: Potenciales de contribución al calentamiento global, relativos al $\mathrm{CO}_{2}$, al que se asigna valor 1 (adaptada de BSI, 2008)

\begin{tabular}{|l|l|r|}
\hline Dióxido de C & CO2 & 1 \\
\hline Metano & CH4 & 25 \\
\hline Oxido Nitroso & N2O & 298 \\
\hline $\begin{array}{l}\text { Sustancias controladas según el protocolo } \\
\text { de Montreal }\end{array}$ \\
\hline CFC-11 CCl3F & \\
\hline CFC-12 & CCl2F2 & 4,750 \\
\hline CFC-13 & CCIF3 & 10,900 \\
\hline CFC-113 & CCl2FCCIF2 & 14,400 \\
\hline CFC-114 & CCIF2CCIF2 & 6,130 \\
\hline CFC-115 & CCIF2CF3 & 10,000 \\
\hline Halon-1301 & CBrF3 & 7,370 \\
\hline Halon-1211 & CBrCIF2 & 7,140 \\
\hline Halon-2402 & CBrF2CBrF2 & 1,890 \\
\hline $\begin{array}{l}\text { Tetracloruro de } \\
\text { carbono }\end{array}$ & CCl4 & 1,640 \\
\hline Bromuro de metilo & CH3Br & 1,400 \\
\hline Metil cloroformo & CH3CCl3 & 5 \\
\hline HdCFC-22 & CHdClF2 & 146 \\
\hline HdCFC-123 & CHdCl2CF3 & 1,810 \\
\hline HdCFC-124 & CHdClFCF3 & 77 \\
\hline HdCFC-141b & CH3CCl2F & 609 \\
\hline PFC-3-1-10 & C4F10 & 725 \\
\hline PFC-4-1-12 & C5F12 & 8,860 \\
\hline PFC-5-1-14 & C6F14 & 9,160 \\
\hline PFC-9-1-18 & C10F18 & 9,300 \\
\hline HdCFC-142b & CH3CClF2 & $>7,500$ \\
\hline HdCFC-225ca & CHdCl2CF2CF3 & 2,310 \\
\hline HdCFC-225cb & CHdClFCF2CCIF2 & 122 \\
\hline HFC-23 & CHF3 & 595 \\
\hline HFC-32 & CH2F2 & 14,800 \\
\hline HFC-125 & CHF2CF3 & 675 \\
\hline HFC-134a & CH2FCF3 & 3,500 \\
\hline HFC-143a & CH3CF3 & 1,430 \\
\hline HFC-152a & CH3CHF2 & 1,470 \\
\hline HFC-227ea & CF3CHFCF3 \\
\hline HFC-236fa & CF3CH2CF3 \\
\hline HFC-245fa & CHF2CH2CF3 \\
\hline HFC-365mfc & CH3CF2CH2CF3 \\
\hline HFC-43-10mee & CF3CHFCHFCF2CF3 & 1,640 \\
\hline
\end{tabular}

\begin{tabular}{|l|l|r|}
\hline \multicolumn{2}{|l|}{ Compuestos perfluorados } & \\
\hline Hexafluoruro de S & SF6 & 22,800 \\
\hline Tetrafluoruro de $\mathrm{N}_{2}$ & NF3 & 17,200 \\
\hline PFC-14 & CF4 & 7,390 \\
\hline PFC-116 & C2F6 & 12,200 \\
\hline PFC-218 & C3F8 & 8,830 \\
\hline PFC-318 & C-C4F8 & 10,300 \\
\hline
\end{tabular}

\begin{tabular}{|l|l|r|}
\hline \multicolumn{2}{|l|}{ Trifluorometil pentafluoruro de S, SF5CF3 } & 17,700 \\
\hline Éteres fluorados & CHF2OCF3 & 14,900 \\
\hline HFE-125 & CHF2OCHF2 & 6,320 \\
\hline HFE-134 & CH3OCF3 & 756 \\
\hline HFE-143a & CHF2OCHdClCF3 & 350 \\
\hline HdCFE-235da2 & CH3OCF2CHF2 & 708 \\
\hline HFE-245cb2 & CHF2OCH2CF3 & 659 \\
\hline HFE-245fa2 & CH3OCF2CHF2 & 359 \\
\hline HFE-254cb2 & CH3OCF2CF2CF3 & 575 \\
\hline HFE-347mcc3 & CHF2CF2OCH2CF3 & 580 \\
\hline HFE-347pcf2 & CH3OCF2-CF2CHF2 & 110 \\
\hline HFE-356pcc3 & C4F9OCH3 & 297 \\
\hline $\begin{array}{l}\text { HFE-449sl (HFE- } \\
\text { 7100) }\end{array}$ & 59 \\
\hline $\begin{array}{l}\text { HFE- } \\
\text { 569sf2(HFE- } \\
\text { 7200) }\end{array}$ & C4F9OC2H5 & 1,870 \\
\hline $\begin{array}{l}\text { HFE-43-10- } \\
\text { pccc124 } \\
\text { (H-Galden } \\
\text { 1040x) }\end{array}$ & CHF2OCF2O- & 13 \\
\hline $\begin{array}{l}\text { HFE-236ca12 } \\
\text { (HG-10) }\end{array}$ & \begin{tabular}{l} 
CH2OCF2- \\
\hline $\begin{array}{l}\text { HFE-338pcc13 (HG-01) } \\
\text { CHF2OCF2CF2OCHF2 }\end{array}$
\end{tabular} \\
\hline \begin{tabular}{l} 
Perfluoropolieters \\
\hline PFPMIECF3OCF(CF3)CF2OCF2OCF3
\end{tabular} & 10,300 \\
\hline Hidrocarburos compuestos & 2,800 \\
\hline $\begin{array}{l}\text { Dimetileter CH3OCH3 } \\
\text { Cloruro de } \\
\text { metileno }\end{array}$ & CH2Cl2 & 1,500 \\
\hline Cloruro de metilo & CH3Cl & \\
\hline
\end{tabular}

A juicio de Carballo et al. (2009) la HdC nace huérfana, sin un concepto claro ni una metodología definida, lo que ha posibilitado que surjan diferentes interpretaciones del indicador. Una de estas diferencias se relacionan con los gases incluidos en el análisis Algunos estudios optan porque la $\mathrm{HdC}$ incluya varios gases de efecto invernadero, expresando la huella del carbono en toneladas equivalentes de $\mathrm{CO}_{2}$ (Dómenech, 2004; CT, 2007; POST 2008; BSI, 2008). Otros prefieren limitarse exclusivamente a un único gas, el $\mathrm{CO}_{2}$ (Wiedmann y Minx, 2008). Los GEl considerados usualmente son los establecidos en el Protocolo de Kioto del año 1997 y consideran los siguientes: dióxido de carbono $\left(\mathrm{CO}_{2}\right)$, metano $\left(\mathrm{CH}_{4}\right)$, óxido nitroso $\left(\mathrm{N}_{2} \mathrm{O}\right)$, hidrofluorocarbonos 
(HFC), hidrocarburos perfluorados (PFC), y hexafluoruro de azufre $\left(\mathrm{SF}_{6}\right)$. Esto no significa que no haya otros gases contaminantes, pero éstos son los criterios actuales; incluso hay criterios de cálculo que no consideran todos estos gases, y esto es también parte del debate.

Todos esto asuntos propician que las definiciones de la HdC propuestas difieran sustancialmente. Global Footprint Network, por ejemplo, define la HdC como "la demanda de biocapacidad precisa para secuestrar, mediante fotosíntesis las emisiones de $\mathrm{CO}_{2}$ procedentes de la combustión de combustibles fósiles" (GFN, 2006). La definición de Carbon Trust es mucho más amplia, al incluir "Ias emisiones totales de gases de efecto invernadero en toneladas equivalentes de un producto a lo largo de su ciclo de vida desde la producción de las materias primas empleadas en su producción, incluso la eliminación del producto acabado" (CT, 2007). Para la aplicación de la HdC a las empresas, algunos estudios recomiendan que la $\mathrm{HdC}$ de bienes y servicios se limite a la estimación de las emisiones directas realizadas por la empresa que los produce. En otros casos, se consideran también las generadas indirectamente, considerando las emisiones producidas en la cadena de proveedores de los que se abastece la empresa para obtener sus insumos (Wiedmann y Minx, 2008). Como se mencionó, uno de los principales obstáculos para avanzar en la implantación de la HdC como parámetro de medición del nivel de contaminación de un proceso productivo, de un producto o de un servicio, es la incertidumbre respecto a su cálculo y la discusión sobre los factores que deben considerarse en su determinación.

Las diversas interpretaciones del concepto de $\mathrm{HdC}$ ha llevado al desarrollo de metodologías de cálculo muy diferentes, situación que genera una excesiva controversia frente a un índice que ya goza de bastante popularidad (Carballo et al., 2009). Algunos autores sostienen que una "huella", por el significado del concepto, debe abarcar todos los efectos que deja tras de sí una actividad. En el caso de una $\mathrm{HdC}$, debe incluir todas las emisiones de gases de efecto invernadero que se pueden asociar directa e indirectamente con una actividad, y por lo tanto el análisis debe abarcar todo el ciclo de vida de un producto o servicio, desde las materias primas e insumos hasta el producto o servicio final. Metodológicamente, esta perspectiva de "todo el ciclo de vida" se ha abordado desde dos direcciones: enfoque de arriba hacia abajo o enfoque corporativo (top-down) y enfoque de abajo hacia arriba o enfoque de producto (bottom-up). El enfoque de arriba hacia abajo parte desde una visión global de una organización, para la obtención de una o más magnitudes específicas de HdC (Minx et al., 2010). El enfoque de abajo hacia arriba realiza un análisis específico de la magnitud de las unidades constituyentes de una organización, evento o proceso y va agregando magnitudes parciales, para obtener una magnitud global de HdC para la unidad en estudio, generalmente un producto o un servicio (Wiedmann, 2009, Minx et al., 2010). .

\section{MÉTODOS TOP-DOWN Y BOTTOM-UP}

Los conceptos top-down y bottom-up (de arriba hacia abajo y de abajo hacia arriba, respectivamente), son estrategias de procesamiento de información características de las ciencias de la información, especialmente en lo relativo al software. Por extensión se aplican también a otras áreas del conocimiento, como la economía, la gestión y la educación. En el análisis topdown (de arriba a abajo) se parte de la visión más amplia del mercado, para ir descendiendo en la cadena de análisis, hasta llegar a la visión más concreta de la empresa objeto de estudio. De un contexto global pasan al valor concreto. En el análisis bottom-up (de abajo a arriba) se comienza el análisis por la parte del mayor detalle posible. Este enfoque, no reniega del conocimiento de la situación macroeconómica, aunque da prioridad al análisis de las empresas como objeto de estudio de oportunidades del mercado. La figura 1 clarifica estos conceptos.

El enfoque bottom-up, esquematizado en la Fig. 1a, orienta al análisis del ciclo de vida de los productos en base al análisis de los procesos, intentando comprender los impactos ambientales de los productos individuales a lo largo de su proceso de transformación, es decir, desde la materia prima hasta su disposición final. Usando los datos específicos del proceso primario y secundario, este enfoque puede lograr resultados con alta precisión para los productos definidos y en cada uno de los procesos. Sin embargo, tiene que establecer un límite del sistema a analizar y por lo tanto, conduce a errores de truncamiento de tamaño desconocido. Debido a los requisitos de datos el análisis es generalmente una tarea que requiere de recursos y de costos más 
elevados que otros métodos (Minx et al., 2010). Como se observa en la figura, la decisión final en el enfoque bottom-up se toma basándose en decisiones individuales y no de globalidad. Una vez analizado el detalle, y en función del interés del analista, éste irá ascendiendo en la cadena del análisis, en sentido contrario al enfoque dado por el análisis top-down de la Fig. 1b.

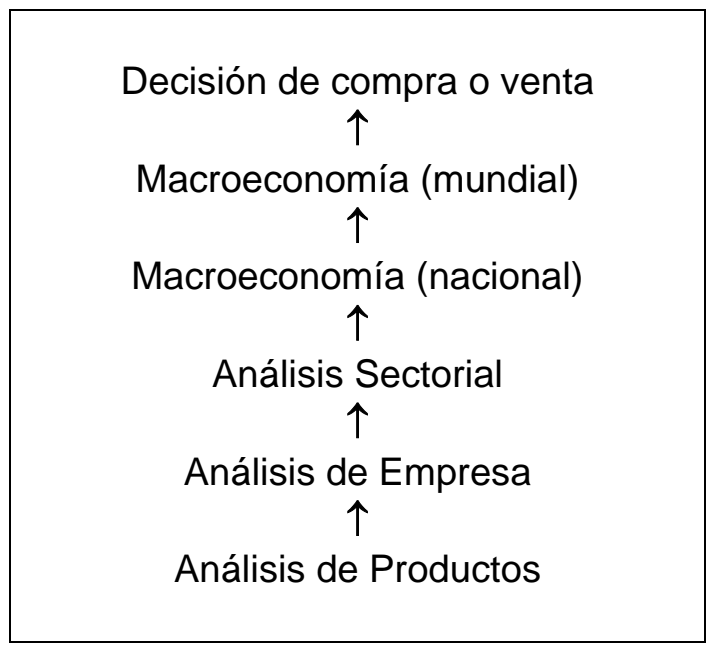

Fig.1a: Análisis Bottom-Up

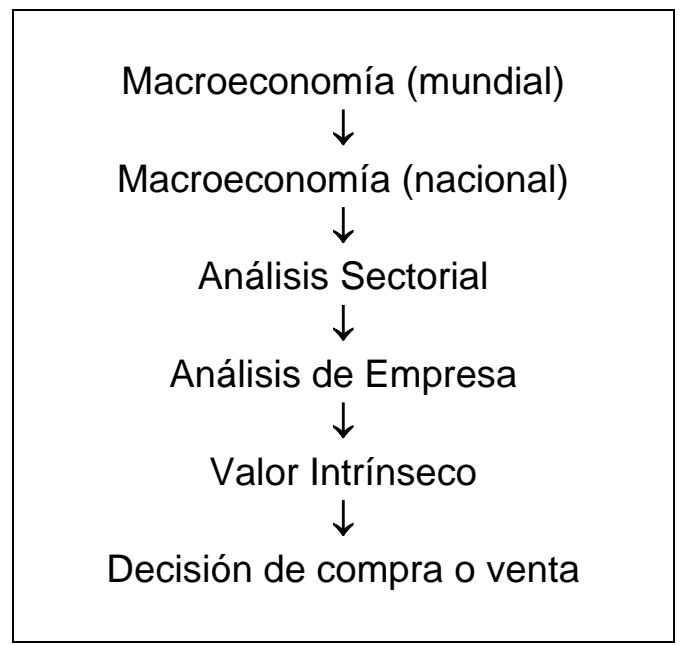

Fig. 1b: Análisis Top-Down

En el contexto de este trabajo estos conceptos son aplicados a la toma de decisiones que pueden ser influenciadas por la acción de un fenómeno externo a las organizaciones productoras de bienes o servicios, como es la inclusión de la huella del carbono. Esta normativa de cuantificación de la HdC está ya siendo incorporada en regulaciones medioambientales locales e internacionales y afectan tanto a la organización como a sus productos. Así, también, afectan la percepción de valor frente a productos o empresas determinando en mayor o menor medida la decisión de compra por parte de los consumidores. Desde el punto de vista del análisis de la HdC el enfoque top-down determina los pasos metodológicos para la obtención de la magnitud y fija el espacio de utilización de los resultados en el orden corporativo repartiendo esta magnitud entre todos los productos o servicios de la organización. El análisis top-down se realiza sobre la base del análisis de entrada-salida de la organización (IOA, Input-Output Analysis), es decir, de la relación de ésta en su conjunto con el medio ambiente o entorno próximo. (Dómenech et al., 2010; Minx et al., 2010). Las figuras 2 y 3 muestran la secuencia de análisis que siguen estos enfoques en la cuantificación de la HdC.

La mejor opción metodológica para la determinación de un valor más real de la HdC parece ser una combinación de ambos enfoques Top-down y Bottom-up. Matthews et al. (2008) proponen determinar la HdC a través de una combinación de ambos métodos, denominado enfoque del ciclo de vida híbrido (Hybrid ACV). Este análisis permite la preservación de los detalles y la precisión de los enfoques de abajo-arriba (bottom-up) en los procesos importantes, mientras que la mayoría de las etapas de producción menos significativas, pueden ser cubiertos por el modelo de entrada y salida (top-down). Dependiendo de la aplicación, las etapas a las que se aplique uno u otro método variará, con el fin de lograr una solución óptima en base a la combinación entre la exactitud, precisión y eficiencia en los costos (Minx et al., 2010). Estas consideraciones son, actualmente, objeto de debate en el proceso de normalización en curso para la HdC. La metodología MC3 V 2.0 (Método Compuesto de las Cuentas Contables versión 2.0), presentada por Dómenech et al. (2010) parece responder de muy buena forma con estos requisitos.

La Tabla 2 muestra una selección de artículos sobre diversas aplicaciones de la HdC. Algunos de los estudios mostrados en la Tabla se han utilizado en los debates políticos, sobre todo en los ámbitos de la política internacional de desarrollo sustentable, la economía del cambio climático, los marcos nacionales de producción, el consumo sostenible, así como las cuestiones locales y regionales de desarrollo. 
Tabla 2: Algunas alternativas de aplicación de la Huella del Carbono presentadas en la literatura (Minx et al., 2010)

\begin{tabular}{|c|c|c|}
\hline Ámbito & HdC de: & Trabajo \\
\hline \multirow{2}{*}{ 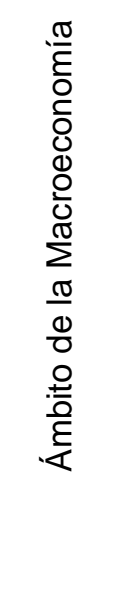 } & Las naciones & $\begin{array}{l}\text { - Balanza comercial o fugas de carbono } \\
\text { - Huella de Carbono de un país específico }\end{array}$ \\
\hline & $\begin{array}{l}\quad \text { Sub } \\
\text { nacionales } \\
\text { regionales o } \\
\quad \text { locales }\end{array}$ & $\begin{array}{l}\text { - Programas de cambio climático para las ciudades y gobiernos locales de las } \\
\text { organizaciones internacionales } \\
\text { - Estandarizar la construcción de la producción local y los inventarios de GEI } \\
\text { basados en el consumo } \\
\text { - Métodos de cálculo de HdC para las pequeñas áreas espaciales (en fase } \\
\text { incipiente de desarrollo) } \\
\text { - Notables excepciones de esta aplicación del método Input Output generalizado }\end{array}$ \\
\hline$\frac{E}{<}$ & $\begin{array}{l}\text { Sectores y } \\
\text { productos }\end{array}$ & $\begin{array}{l}\text { - Determinación de emisiones de GEI directos e indirectos asociados con la } \\
\text { producción sectorial y actividades de consumo } \\
\text { - Análisis de puntos críticos de carbono sectoriales } \\
\text { - Identificación de áreas prioritarias de investigación de cambio climático y } \\
\text { política } \\
\text { - Modelos híbridos de enfoque (ACV- Input- Output) para análisis del ciclo de vida } \\
\text { de los productos individuales o la evaluación comparativa de las empresas } \\
\text { individuales en contra de la media del sector }\end{array}$ \\
\hline$\downarrow$ & $\begin{array}{l}\text { Cadenas de } \\
\text { suministro }\end{array}$ & $\begin{array}{l}\text { - Rastreo de fuentes de fuentes de emisión asociados a una cadena de } \\
\text { suministro particular de una demanda final sectorial de } 57 \text { sectores y } 87 \\
\text { regiones del mundo }\end{array}$ \\
\hline \multirow[b]{2}{*}{ 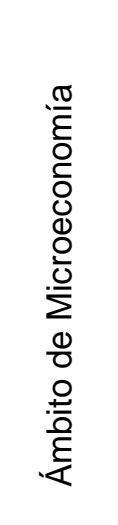 } & $\begin{array}{c}\text { Organizacion } \\
\text { es }\end{array}$ & $\begin{array}{l}\text { - Contabilidad de las organizaciones } \\
\text { - La vinculación de entrada y salida modelos generalizados con las cuentas } \\
\text { financieras de una organización en un marco híbrido }\end{array}$ \\
\hline & $\begin{array}{l}\text { Patrones de } \\
\text { consumo y } \\
\text { estilos de } \\
\text { vida }\end{array}$ & $\begin{array}{l}\text { - Patrón de gastos de un grupo de personas u hogares con un conjunto bien } \\
\text { definido de características socio demográficas } \\
\text { - Estudios comparativos para identificar el consumo de paquetes y/o grupos de } \\
\text { estilo de vida con el potencial de ahorro de carbono } \\
\text { - Estudios para identificar los factores subyacentes que afectan las emisiones } \\
\text { - Estudios sobre las presiones generado por cambiar estilos de vida o la } \\
\text { demografía } \\
\text { - Detalles metodológicos necesarios para una cabal comprensión de los estudios } \\
\text { de Huella de Carbono de estilos de vida y patrones de consumo }\end{array}$ \\
\hline
\end{tabular}

\section{Enfocue Top -Down $\rightarrow$ (Alcance 1 +Alcance 2 ) +Alcance 3 = HdC Corporativo}

ALCANCE 2 ALCANCE 1 ALCANCE 3

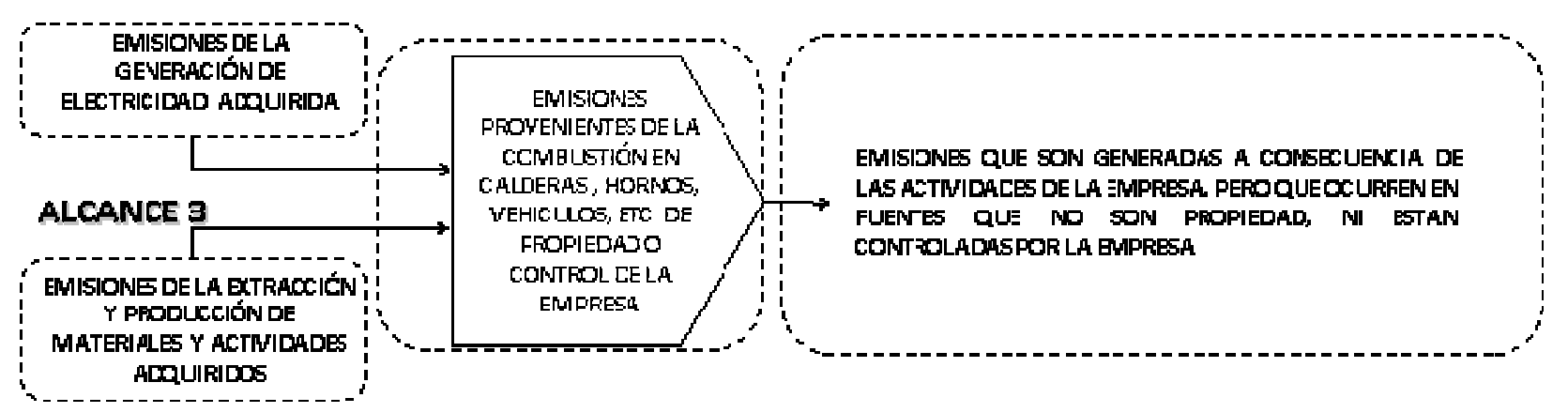

Fig. 2: Enfoque de arriba-hacia-abajo para el análisis de emisiones y HdC 
Enfoque Bottom $-U_{p}>$ Emisiones etapa $1+\ldots+$ Emisiones etapa $n=\mathrm{HdC}$ producto

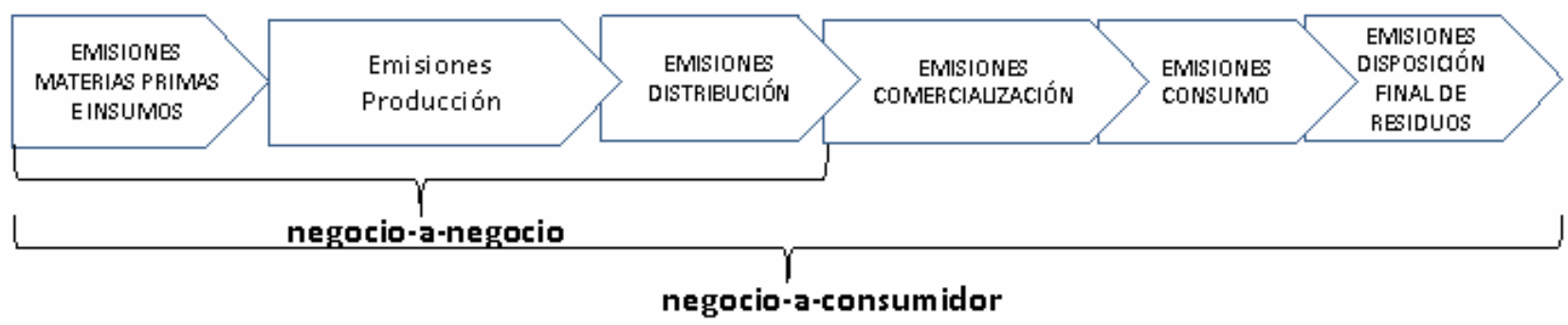

Fig. 3: Enfoque de abajo-hacia-arriba para el análisis de emisiones y HdC

\section{MÉTODOS ACTUALES}

Existen cuatro métodos principales presentados en la literatura para el cálculo de la HdC en empresas, organizaciones, servicios, procesos y productos. Estos métodos utilizan un acercamiento muy similar para la obtención de datos como es el análisis de ciclo de vida del producto. Una vez que se esquematiza el ciclo de vida del producto, desde que se extraen las materias primas necesarias para su fabricación hasta el lugar de su disposición final, cada método usa un enfoque diferente. La primera diferencia tiene lugar en la limitación del alcance de la empresa, es decir hasta dónde las emisiones de otros procesos influyen en el proceso en estudio. Una vez que se tiene el proceso bien delimitado, se extraen datos diferentes del proceso, para cada método de determinación. La Tabla 3 resume los cuatro métodos principales disponibles para cuantificar la HdC.

\section{Protocolo de Gases Efecto Invernadero}

El Protocolo de Gases Efecto Invernadero (GEI) fue implementado en el 2001 por el Consejo Mundial de Negocios por el Desarrollo Sustentable (World Business Council for Sustainable Development, WBCSD) y por el Instituto de Recursos Mundiales (World Resources Institute, WRI) que tiene como meta el establecimiento de bases para la contabilización de emisiones de los GEI. Es fruto de una colaboración multilateral entre empresas, organizaciones no-gubernamentales y gobiernos (WRI, 2004). Esta iniciativa cuenta con el apoyo de la Agencia de los Estados Unidos para el Desarrollo Internacional (United States Agency for International Development, USAID) y la Agencia de Protección Ambiental de los Estados Unidos (United States Environmental Protection Agency, USEPA). El protocolo de GEI es un marco metodológico general que da pautas de trabajo para la determinación de herramientas (software) de cálculo de emisiones de GEI. El protocolo GEI ha logrado un alto nivel de reconocimiento a escala mundial y aparece como la principal referencia, junto con los estándares ISO 14064. Además de constituirse como referente en términos de lineamientos generales, el protocolo GEI también ha desarrollado un conjunto de herramientas (software) para el cálculo de la HdC inicialmente de empresas. La popularidad y el reconocimiento del protocolo GEI, y por supuesto el carácter de gratuidad de las aplicaciones, ha concretado el éxito y alta demanda de ellas (Pandey et al., 2010).

\section{Balance de Carbono}

La metodología y a la vez herramienta Balance de Carbono, conocida internacionalmente como Bilan Carbone, fue desarrollada por la Agencia del Medio Ambiente y Energía de Francia, ADEME, en el año 2002 (BC, 2011). La herramienta fue elaborada específicamente para convertir datos relativos a las actividades productivas (como el consumo de energía, la cantidad de camiones y la distancia manejada, las toneladas de acero adquiridas, entre otras) en emisiones, de forma rápida, usando factores de emisión. El método considera la contabilización de emisiones directas e indirectas de los GEI, relacionadas con las actividades industriales, empresariales, y de otras asociaciones y entidades administrativas. Permite clasificar las emisiones según fuente, siendo la base de la herramienta, una planilla Excel que calcula las emisiones asociadas a cada actividad de un proceso (ADEME, 2011; Jancovici, 2003). 


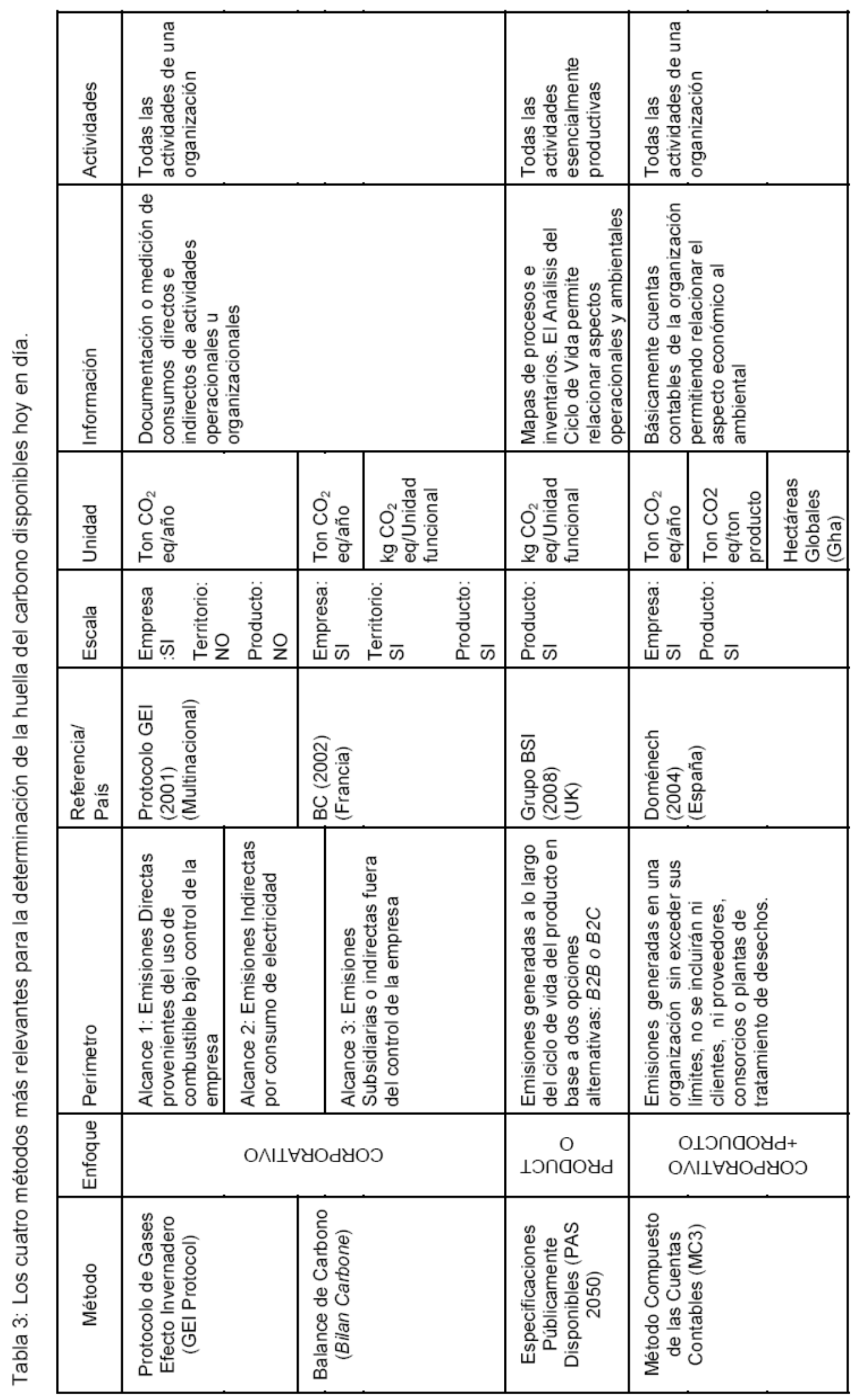


El Bilan Carbone se caracteriza por una visión general muy completa, por lo que, a través de sus distintos módulos, permite trabajar a nivel de empresas y eventos, pero también de territorios y productos. Sus primeras versiones fueron implementadas en el año 2004 y responde a los requisitos de los marcos metodológicos ISO 14.064 y el protocolo de gases efecto invernadero (GEI). Con el apoyo de subvenciones estatales francesas otorgadas a las entidades que utilizan este método para medir las emisiones de los GEl el Bilan Carbone ${ }^{\circledR}$ se transformó en la referencia metodológica en Francia para las empresas, las personas y las colectividades territoriales. (BC, 2011).

\section{Especificaciones Públicamente Disponibles, PAS 2050}

El método de las Especificaciones Públicamente Disponibles (Publicly Available Specification), llamado PAS 2050, fue elaborado en el año 2007 por el Instituto Británico de Estandarización (BSI, 2008), con el apoyo del Consorcio del Carbono (Carbon Trust) y el Depto. para el Ambiente, la Alimentación y Asuntos Rurales (DEFRA), ambos organismos del gobierno inglés. El método está enfocado al cálculo de las emisiones de productos y servicios y responde a las normativas ISO 14040 referido al Análisis del Ciclo de Vida y 14067 Huella de carbono para productos (actualmente en preparación), así como a las recomendaciones del Protocolo GEl. La metodología PAS 2050 define inicialmente las fuentes de emisiones consideradas, además de seis grandes bloques de actividades, cuyas emisiones deben ser consideradas en la estimación del ciclo de vida de bienes y servicios (ver Fig. 2). Con la enumeración de las fuentes de emisiones consideradas, realiza una acotación general del ámbito al que se aplica el indicador. Los gases considerados en el cálculo de la $\mathrm{HdC}$ y sus efectos potenciales en el calentamiento global son mostrados en la Tabla 1. En la Tabla el potencial del $\mathrm{CO}_{2}$ es definido como 1.0 y los valores de los otros gases son referidos a este valor de referencia. El potencial de contribución al calentamiento global (Global Warming Potential, GWP) es una medida útil para comparar los efectos reales de las emisiones de cada gas. Los valores deben ser usados con discreción ya que no consideran algunos factores importantes de comparación como es por ejemplo la vida media de las especies mostradas. (BSI, 2008).

El año 2010, el Instituto Británico de Estandarización presentó la PAS 2060, una metodología similar a la PAS 2050 pero dedicada al cálculo de las emisiones de organismos (administración, empresas, sitio de producción), colectividades territoriales y particulares. Esta metodología sigue las normativas del ISO y del protocolo GEI (CEPAL; 2009). Similar a la PAS 2050, la PAS 2060 es una guía metodológica que describe paso a paso los criterios a determinar y considerar para cuantificar la HdC. La PAS 2060 constituye una poderosa herramienta para las buenas prácticas de compensación de emisiones no reductibles de GEI, y está orientada a operadores que buscan ser neutros en carbono (BSI, 2010).

\section{Método Compuesto de las Cuentas Contables}

El Método Compuesto de las Cuentas Contables (MC3) ha sido desarrollado por Doménech y colaboradores a partir del año 2004 (Dómenech et al., 2010). En este método la información fluye directamente de una organización a otra sin necesidad de contar con la colaboración de clientes o proveedores de la cadena de suministro para calcular la huella. Toda la huella de productos pasa de una entidad a otra, acumulándose progresivamente, y el estudio de huella se limita siempre a una única entidad. De este modo, el alcance organizacional queda perfectamente delimitado en todo momento. Por eso, MC3 nunca incorpora el uso del producto por parte de un consumidor, o la destrucción del mismo al final de su vida útil, ya que esto queda fuera del alcance organizacional.

Comparado con las otras metodologías, esta propuesta tiene algunas ventajas: 1) presenta un "enfoque a la organización", lo cual permite el ecoetiquetado de la organización y de sus productos o servicios con la misma metodología; 2) permite expresar el indicador tanto en toneladas de $\mathrm{CO}_{2}$ (huella de carbono) como en hectáreas de terreno bioproductivo (huella ecológica); 3) permite extraer los datos de entrada de las cuentas contables, lo cual permite a su vez, incorporar todas las fuentes posibles de emisión de carbono, permitiendo así la 
comparabilidad; 4) finalmente, el ciclo de vida previo se incorpora con los productos o consumos de entrada, con lo cual no es necesaria la colaboración directa de clientes o proveedores; la información fluye automáticamente a lo largo de la cadena de suministro.

Puesto de una manera simple la $\mathrm{HdC}$ en cualquiera de los métodos descritos arriba es calculada adicionando mediante algún modelo matemático los variados factores que inciden en la producción de GEI. Un modelo simple, para explicar la forma de cálculo, es la suma ponderada de los diversos factores, como se describe en la ecn. (1)

$\mathrm{HdC}=\sum \mathrm{x}_{\mathrm{i}} \mathrm{y}_{\mathrm{i}} \mathrm{F}_{\mathrm{i}}$

En esta ecuación general, $x_{i}$ e $y_{i}$ son factores de conversión y equivalencia que dependerán del tipo aplicación ( $\mathrm{HdC}$ del producto, de una empresa, de un servicio, de una vivienda) y magnitud (local, regional, o de país) y de la influencia de la variable $F_{i}$ (transporte en avión, o auto, uso de carbón o gas natural, emisiones directas o indirectas, kilómetros recorridos, entre muchos otros).

En una aplicación doméstica, por ejemplo en el cálculo de la HdC anual de una familia, el factor $F_{1}$ puede ser la electricidad usada, $F_{2}$ la cantidad de autos que tiene la familia o los kilómetros recorridos, $F_{3}$ los metros cuadrados construidos, $F_{4}$ el gasto en supermercado, $F_{5}$ los viajes realizados y las distancias recorridas en avión, entre variados otros factores. En el cálculo de la $\mathrm{HdC}$ de un producto, el factor $F_{1}$ puede ser por ejemplo el tipo y tamaño de envase, $F_{2}$ el origen de las materias primas, $F_{3}$ tipo de producto analizado, $F_{4}$ el tipo de proceso al que fue sometido, $F_{5}$ el tipo de energía utilizada en su manufactura, entre otros. En una aplicación industrial por ejemplo, el factor $F_{1}$ puede ser la cantidad de combustible de tipo $A$ que usa la empresa, $F_{2}$ la cantidad de combustible de tipo $\mathrm{B}, \mathrm{F}_{3}$ la electricidad usada, $\mathrm{F}_{4}$ la cantidad de acero usado en el proceso, $\mathrm{F}_{5}$ la cantidad de plásticos y materiales no degradables que consume la empresa, $F_{6}$ los metros cuadrados construidos, $F_{7}$ la cantidad de personas que trabajan en la empresa, entre otros posibles factores. La autora Alexandra Shimo-Barry (2008) en su libro "La ecuación ambiental", propone un simple método para calcular la HdC doméstica. La autora define 8 variables (Fi) y sus respectivos factores de equivalencia y conversión. La Tabla 4 muestra las variables y los factores de conversión, para la ecuación 1 . El resultado es la $\mathrm{HdC}$ en libras de $\mathrm{CO}_{2}$ por año. El promedio en los Estado Unidos es de 16 a 22 mil.

Tabla 4: Variables y factores de equivalencia para calcular la HdC doméstica (Shimo-Barry, 2008)

\begin{tabular}{|c|l|l|}
\hline$N^{0}$ & Variable $\mathrm{F}_{\mathrm{i}}$ & $\mathrm{x}_{\mathrm{i} \times \mathrm{yi}}$ \\
\hline 1 & Gasto mensual en electricidad en US\$ & 105 \\
\hline 2 & Gasto mensual en gas en US\$ & 105 \\
\hline 3 & Gasto mensual en gasolina gas en US\$ & 113 \\
\hline 4 & Millas recorridas en su auto al año & 79 \\
\hline 5 & Número de vuelos de menos de 4 horas por año & 1,100 \\
\hline 6 & Número de vuelos de más de 4 horas por año & 4,400 \\
\hline 7 & Recicla papel de diario y similares & Si es NO agregue 184; Si es SI no agregue nada \\
\hline 8 & Recicla envases aluminio y estaño & Si es NO agregue 166; Si es SI no agregue nada \\
\hline
\end{tabular}

Para el cálculo de la HdC global, Ewing et al. (2010) presentan esta ecuación:

$\mathrm{HdC}=\frac{\mathrm{P}_{\mathrm{C}}\left(1-\mathrm{S}_{\mathrm{O}}\right)}{\mathrm{Y}_{\mathrm{C}}} * \mathrm{EQF}$ 
Aquí, $\mathrm{P}_{\mathrm{C}}$ es la producción anual de emisiones de $\mathrm{CO} 2, \mathrm{~S}_{\mathrm{O}}$ es la fracción de emisiones antropogénicas, esto es producidas por las actividades humanas, que es secuestrada por los océanos en un año, $\mathrm{Y}_{\mathrm{C}}$ es la cantidad total de carbono absorbido por hectárea de bosque disponible, y EQF es un factor de equivalencia para el país, el año, y el tipo uso de tierra. La producción anual de emisiones $\mathrm{P}_{\mathrm{c}}$ es calculada adicionando mediante algún modelo matemático los varios factores que inciden en la producción de GEl, similar al modelo de la ecn. (1).

Sobre qué valores de los factores de conversión y equivalencia y cuales variables $F_{i}$ considerar en el cálculo de la HdC no hay consenso aún en instituciones, empresas y en la academia. Esto ha llevado a que no se tenga aún un método aceptable y general para determinar y comparar la HdC de productos, servicios, viviendas, y empresas. La solución a estas diferencias y cuestionamientos a la utilidad de la HdC como indicador de sustentabilidad ambiental, cuando se trata de productos, podría tener alguna solución con la aprobación del estándar ISO 14067 en su texto refundido de sus normas ISO 14067-1 e ISO 14067-2.

\section{CONCLUSIONES}

Los antecedentes de la literatura muestran que el tema está llegando de a poco a los usuarios y a quienes provocan nuestros problemas ambientales (nosotros mismos).

Hay consenso general de que a mayor concentración de gases con efecto invernadero se producirá mayor aumento en la temperatura en la Tierra. A partir de 1979 los científicos comenzaron a afirmar que un aumento al doble en la concentración del $\mathrm{CO}_{2}$ en la atmósfera supondría un calentamiento medio de la superficie de la tierra de entre 1,5 y $4,5^{\circ} \mathrm{C}$.

La HdC perfila como un indicador capaz de sintetizar los impactos provocados por las actividades del hombre en el entorno, medido en términos de emisiones de GEI. Por lo tanto, la HdC representa una poderosa herramienta de gestión y un estímulo para adoptar una estrategia para el logro de la sustentabilidad de las organizaciones.

Sobre qué valores de los factores de conversión y equivalencia, cuales variables $F_{i}$ y que alcances considerar en el cálculo de la $\mathrm{HdC}$, no hay consenso aún en instituciones, empresas y en la academia. Por lo tanto, no existe aún un método aceptable y general para determinar y comparar la HdC de productos, servicios, viviendas y empresas.

Se hace necesario avanzar en la difusión de las bases conceptuales involucradas en la HdC, en todos los actores de la sociedad de manera de facilitar la discusión y búsqueda de acuerdos

La solución a las diferencias y cuestionamientos a la utilidad de la HdC como indicador de sustentabilidad ambiental, cuando se trata de productos, podría tener alguna solución con la aprobación del estándar ISO 14067 en su nuevo texto refundido de las normas ISO 14067-1 e ISO 14067-2.

\section{AGRADECIMIENTOS}

Los autores agradecen el apoyo prestado por la Universidad de La Serena y del Centro de Información Tecnológica (Chile) para la realización de este trabajo. CE agradece también al Depto. Adm. Empresas y Gestión Económica de Rec. Naturales, de la Universidad de Lleida (España).

\section{REFERENCIAS}

Ademe (l'Agence de l'Environnement et de la Maîtrise de l'Energie). Bilan Carbone ${ }^{\circledR}$, Entreprises et Collectivités. Guide méthodologique, version 6.1, objectifs et principes de comptabilisation, (2010).

Bilan Carbone (BC). Disponible en http://www2.ademe.fr/servlet/KBaseShow?sort=-

1 ycid=96ym=3ycatid=15730. Acceso en 20.01 (2011). 
Bristish Standards Institute (BSI). PAS 2050: Specification for the assessment of the life cycle greenhouse emissions of goods and services, (2008) Disponible en http://www.bsigroup.com/en/ Standards-and-Publications/Industry-Sectors/Energy/PAS-2050. Acceso en 20.12. (2010).

Bolin, B., B.R. Döös, J. Jäger y R.A. Warrick. The greenhouse effect, climatic change and ecosystems, International Council of Scientific Unions, Paris (France). Scientific Committee on Problems of the Environment, p. 541, (1986).

Carballo, A., J.L. Doménech y M.C. García. "El ecoetiquetado en base a la huella ecológica y del carbono: una herramienta de marketing verde". UAI Sustentabilidad, 3(7), 1-2 (2009).

CT, Carbon Trust. Carbon footprint measuring methodology. The Carbon Trust. Londres, UK, (2007).

Comisión Económica Para América Latina e el Caribe (CEPAL). Cambio climático y desarrollo en América Latina y el Caribe: una reseña (2009).

Daly, H.E. On Economics as a Life Science. Journal of Political Economy, 76, 392-406 (1968).

De La Torre, A., P. Fajnzylber y J. Nash. Desarrollo con menos carbono: respuestas latinoamericanas al desafío del cambio climático. Banco Central, Washington D.C., USA, (2009).

Dómenech, J. L., A. Carballo, L. Jiménez y J.L. De La Cruz. Estándares 2010 de Huella de Carbono MC3. Conama10 Congreso Nacional de Medio Ambiente, 11,2010. Anais. Madrid, España (2010).

Dómenech, J.L. Huella ecológica portuaria y desarrollo sostenible. Puertos, 114, 26-31 (2004).

Ewing B., A. Reed, A. Galli, J. Kitzes y M. Wackernagel. Calculation Methodology for the National Footprint Accounts, 2010 Edition. Oakland: Global Footprint Network (2010).

EPA, United States Environmental Protection Agency, www.epa.gov (2011).

Global Footprint Network (GFN). Ecological footprint and biocapacity. Technical notes: 2006 edition, Oakland, CA, USA. (2006).

Hertwich, E. y G. P. Peters. Carbon Footprint of Nations: A Global, Trade-Linked Analysis. Environmental Science \& Technology, .43, 6414-6420 (2009).

IPCC, Intergovernmental Panel On Climate Change, United Nations Environment Programme (UNEP) and the World Meteorological Organization (WMO), http://www.ipcc.ch (2011).

Jancovici J. M. Un outil pour connaître les émissions de gaz à effet de serre d'une entreprise ou administration : le "bilan carbone" de l'ADEME, 2003. Disponible en http://www.manicore.com/ missions/bilan_carbone.html. Acceso en 01.03 (2011).

Leontief, W. y D. Ford. Air Pollution and the Economic Structure: Empirical Results of Input-Output Calculations. Paper presented at the Fifth International Conference on Input-Output Techniques North Holland Pub. Co Geneva, Switzerland (1971).

Leontief, W. Environmental Repercussions and the Economic Structure: An Input-Output Approach. Review of Economics and Statistics, 52, 262-271 (1970).

Matthews, H. S., C.T. Hendrickson y C.L. Weber. The Importance of Carbon Footprint Estimation Boundaries. Environ. Sci. Technol. 42(16), 5839-5842 (2008).

Minx, J.C., G.P. Peters, T. Wiedmann y J. Barrett. GHG Emissions in the Global Supply Chain of Food Products. The 2008 International Input-Output Meeting on Managing the Environment (IIOMME), Seville, Spain, July 9-11 (2008).

Minx, J.C., T. Wiedman y otros 14 autores. Input-Output analysis and carbon footprinting: an overview of applications. Economic Systems Research, 21(3), 187-216 (2010). 
Pachauri, R. K. y A. Reisinger (editores). Cambio climático 2007: Informe de síntesis. Contribución de los Grupos de trabajo I, II y III al Cuarto Informe de evaluación del Grupo Intergubernamental de Expertos sobre el Cambio Climático. Ginebra: Cambridge University Press, UK (2007).

Padgett, P., A. Stenemann, J. Clarke y M.A. Vanderbergh. A Comparison of Carbon Calculators, Environmental Impact Assessment Review, 28, 106-115 (2008).

Pandey, D. M. Agrawal y J. Pandey. Carbon footprint: current methods of estimation. Environmental Monitoring and Assessment, 178(1-4), 135-160 (2010).

Plasmann, K, A. Norton, N. Attarzadech, M.P. Jensen, P. Brenton y G. Edwards-Jones. Methodological complexities of product carbon footprinting: a sensitivity analysis of key variables in a developing country context. Env. Sci. \& Police, 13, 393-404 (2010).

POST, Parliamentary Office of Science and Technology, note 268, London, UK. October, 2006. Disponible en: http://www.parliament.uk/documents/upload/postpn268.pdf. Acceso en 12.10 (2008).

Schneider, H. y J.L. Samaniego. La huella del carbono en la producción, distribución y consumo de bienes y servicios, documentos de proyectos, $N^{\circ} 298$, Santiago de Chile, Comisión Económica para América Latina y el Caribe (2009).

Shimo-Barry, A. y C.J. Maron. The Environment Equation, 100 factors that can add to or subtract from your total carbon footprint. Adams Media Corporation, Avon, Massachusetts-USA (2008).

Stern, N. Stern Review on the Economics of Climate Change, 2006. Disponible en www.sternreview.org.uk. Acceso en 20.02 (2011).

Valderrama, J.O., A. Espíndola y R. Quezada. Huella de Carbono, un Concepto que no puede estar Ausente en Cursos de Ingeniería y Ciencias. Form. Univ., .4(3), 3-12 (2011).

Victor, P. A. Pollution: Economy and Environment George Allen and Unwin, Oxford (1972).

WRI, Resources Institute), The Greenhouse Gas Protocol, A Corporate Accounting and Reporting Standard-Marzo 2004. Disponible en http://www.ghgprotocol.org/files/ ghgp/public/ghg-protocolrevised.pdf. Acceso en 20.04 (2011).

Wiedmann T. Carbon Footprint and Input-Output Analysis - An Introduction, Economic Systems Research, 21, 175-186 (2009).

Wiedmann, T. y J. Minx. A Definition of Carbon Footprint In: C. C. Pertsova, Ecological Economics Research Trends, n. 1, p. 1-11, Nova Science Publishers, Hauppauge NY, USA (2008).

WBCSD, World Business Council For Sustainable Development. Energy, Climate Change and Future Framework Conditions: A new approach is needed, Report Dec. 8, (2004). 